\title{
Adaptive Customer Profiles For Context Aware Services in a Mobile Environment
}

\author{
Mike Radmacher \\ Chair of Mobile Business and Multilateral Security, \\ Gräfstr. 78, 60054 Frankfurt am Main, Germany, \\ mike.radmacher@whatismobile.de, \\ WWW home page: http://www.whatismobile.de
}

\begin{abstract}
Mobile data communication generated 10 percent of the overall data revenue in Germany and between 2-3 percent worldwide [29] in 2004. This development is contradictory compared to the high investment (91.5 billion $€$ ) in networks and licenses that supported the UMTS infrastructure and thereby the mobile internet. An advertised-based revenue model [10] addresses an opportunity to increase the mobile data communication. Mobile customers and advertisers are matched based on the customer's current situation (location, time and interests). Precise customer profiles, as a requirement to overcome the information overflow, and to enable a multilateral economically reasonable matching are indispensable but the profile's quality is not given in reality. Without precise customer profiles there is no matching. With situation adaptive customer profiles the profile's quality is increasing. Its design, realization and integration into the mobile operator's infrastructure are the aim of this paper.
\end{abstract}

\section{Introduction}

With the beginning of the 90 's the prerequisites in order to establish mobile data communication as an additional service alongside voice communication were given. 20.3 billion short messages were sent in Germany in 2005 [5]. The mobile data communication generated only 10 percent of the overall data revenue in Germany. This development is contradictory compared to the high investment $(91.5$ billion $€)$ in networks and licenses that supported the UMTS infrastructure and thereby the mobile internet [10].

Furthermore, the penetration of the mobile market in Germany reached nearly $102.3 \%$ in 2006 [5] and implies a high amount of customers under multiple contracts (pre and post paid) in comparison to new customers. The stress of competition is increasing. New business models as e.g. an advertising-based revenue model shown in [10], promote mobile data communication. Mobile network operators take part in the market in an

Please use the following format when citing this chapter:

Radmacher, M., 2007, in IFIP International Federation for Information Processing, Volume 251. Integration and Innovation Orient to L-Society Volumel, Wang, W. (Lds), (Boston: Springer), pp. 390-399. 
additional function as context provider. Unique features of mobile communication (e.g. location and time dependency, identity and context reference, opportunities of interaction) influence e.g. the usage of mobile services. Advertisers gain a communication channel to a customer after an opposite matching of interest. Merging customer's and advertiser's interests on the one hand, and reducing the information flow based on the high amount of information, products, services on the internet on the other hand, requires a precise image of a customer. A question that includes some of the main aspects is given [31] "To give customers exactly what they want, you first have to learn what that is. It sounds simple, but it's not". Reducing the information overflow on the fixed internet is addressed by individualization strategies as for instance recommendation techniques [24]. Amazon is using recommendation techniques typically for products while Google is using them for individualized information providing, but any kind of recommendation based on individualization is strongly dependent on an accurate customer profile.

The mobile market is seeking for differentiation by individualization or personalization. Following this path customer profiles are one key, but in most cases there is less knowledge about customer's preferences. Customers are not interested in sharing their interests or not completely sure about them [21,22]. Checking the quality of customer inputs [21] as a question of truthful statements is missing. Some disclosed personal preferences are strongly related to the benefit the customer is looking for. 94 percent of all internet customers don't disclose personal information at all. 40 percent of them disclose untruthful information [21]. Self-adaptive customer profiles can counteract this development.

This paper aims at presenting first ideas to construct a framework for designing adaptive customer profiles that means using recommender techniques to enhance customer profiles as one of the main problems instead of providing e.g. products recommendations. Furthermore it is addressing the mobile environment and presents a guideline on how to integrate adaptive customer profiles in the existing mobile network operator's infrastructure (e.g. mobile portal). Adaptive customer profiles can provide more accurate personalization and in addition advance current and future recommender mechanisms as well as individualized communication between the advertiser and customer as shown in [10]. By generating knowledge within the own mobile network, switching costs are produced which can also provide the necessary differentiation in the mobile market.

This paper is structured as follows: section 2 discusses the underlying research approach, related to the design research. Section 3 gives the first design of the framework for adaptive customer profiles, while section 4 presents a customer profile designed for a mobile environment. Section 5 visualizes a first guidance on how to integrate the mobile customer profile in the mobile network operator's infrastructure. Section 6 highlights questions of data protection and finally section 7 sums up the contribution of what we learned, and how identified questions can be addressed in more detail in future.

\section{Research Approach}

Based on design research, $[14,27,28]$ the self-created procedure in order to answer the upcoming research questions is visualized in figure 2 . Every single stage is related to the design research approach by [28] 


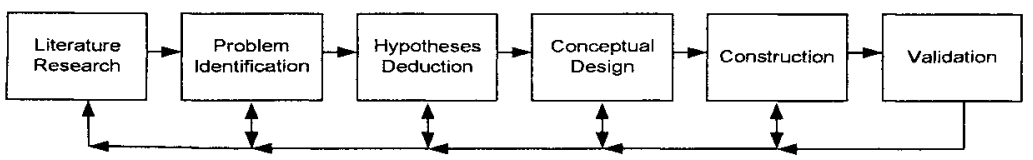

Fig. 1. Research progress related to design reseach

The procedure is separated into six stages and starts with the literature research, followed by problem identification (awareness of a problem). Hypotheses deduction and a first conceptual design (suggestions) are part of the third and forth stage. Stage five is about the construction (development) of a specific artifact and the procedure concludes with the evaluation (evaluation) in stage six.

Three main and three sub questions describe the research questions that are targeted by this paper. (Question1, also motivation) What kind of benefit do adaptive customer profiles generate from the perspective of a service provider and demander? (Question2) How to design adaptive customer profiles in a mobile environment? (Question3) How to integrate adaptive customer profiles into an existing infrastructure of a mobile network operator? (Related to Question1) How do customer profiles influence individualized service usage? (Related to Question2) What are the elements of an adaptive customer profile? (Related to Question3) How to activate still existing knowledge in companies in order to fill these profiles?

Every research starts with literature research. The areas individualized sales approach, techniques and algorithms of recommender systems and self-learning mechanism were investigated in order to profile customers in the best possible way. According to the results of the literature research customer profiles already exist in different ways. In [19] it says customer profiles were suggested as an improvement for a variety of applications. From query enhancement [16] and digital libraries [2], to the personalization of websites [12] and enhanced interpersonal communication [18]. Current trends are for the integration of customer profiling in the delivery of services for an aware environment such as family interactive TV [13], exhibitions [17], filtering of news messages [6, 23] and analyzing customers behavior while visiting internet sites $[25,26]$. In [19] you will also find an overview of existing patents according to customer profiles which do not have any relation to a mobile environment and its particularities. A framework of how to design adaptive customer profiles including mobile aspects was not found through literature research as well as the idea of using recommender techniques to enhance existing customer profiles in order to advance the baseline for recommendations.

The second stage is about the problem identification. The introduction already points out that the information overflow from a customer perspective [20] and the sales approach from an advertiser perspective [9] are still not optimized fields by research. By using adaptive customer profiles, a reduction of search costs both on the customer and advertiser side can lead to differentiation and separation in the mobile commerce against the competition. The aim is to set up a framework for designing adaptive customer profiles based on existing techniques, algorithm and mechanism in order to construct a profile extensible by recommendation. Finally the integration into a mobile network operator's infrastructure is necessary.

The following hypotheses state what kind of benefits an adaptive customer profile should generate. These have to be evaluated in one of the next steps. (Hyp 1) More precise customer profiles should optimize the sales approach. (Hyp2) Based on adaptive customer profiles the current recommendation mechanism might generate better recommendations. (Hyp3) An integration of adaptive customer profiles into the mobile network operator's infrastructure should support the differentiation against competition. 
The conceptual design as fourth stage comprises three artifacts. (Artifactl) A framework for designing an adaptive customer profile. It is a kind of procedure and points out how to design an adaptive customer profile independent of usage driven by the idea to enhance profiles by recommendations. (Artifact2) The development of adaptive customer profile designed for a mobile environment. (Artifact3) A guideline on how to integrate the adaptive customer profile into a mobile network operator's infrastructure.

The construction includes three artifacts named above. The validation should be performed by using the architecture analysis as one method of the design research approach [28] which can test the integration e.g. into an existing infrastructure of a mobile network. Furthermore a case study is planned to get an impression of the quality improvement of recommendations. The field of transaction costs probably leads to a design recommendation to visualize the economic benefits adaptive customer profiles are providing.

\section{Framework Development}

Based on the literature research the following framework is a first approach combing existing, different algorithms, techniques and methods of knowledge management, recommender and self-learning systems in order to recommend profile enhancements for customer profiles. Figure 2 shows the framework, separated into two parts - customer and intermediary. The customer profile (part of the customer) is an image of customer specific information. A detailed description is given in section 4 .

The intermediary (e.g. a mobile network operator) includes several components as databases, a knowledge discovery process, a recommender process, a profile generation process and a feedback process for gathering customer information, and to create an image of the customer. These components are a combination of different approaches, the literature research discovered, and additional components derived from knowledge in the area of mobile commerce.

In several distributed databases, usage specific information is saved, e.g. a service password and customer name, products bought of late and websites visited. All this information, pseudonymous stored, leads to a better understanding of what the customer is looking for.

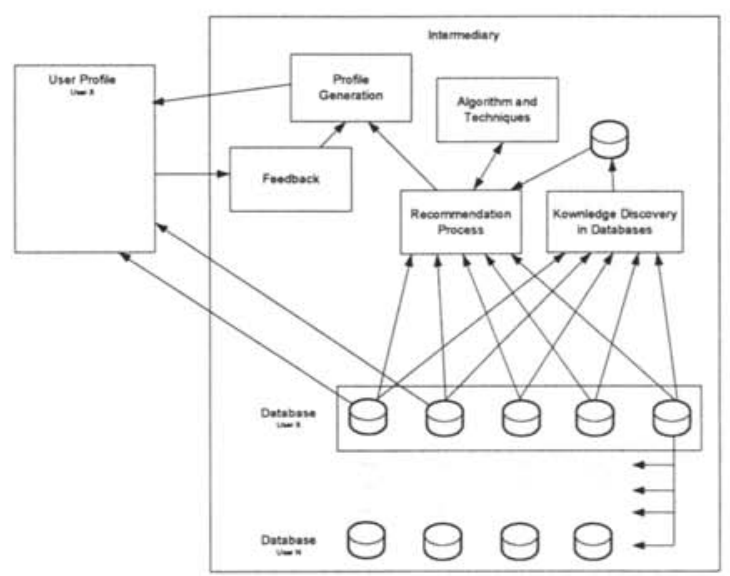

Fig. 2. Framework for adaptive customer profiles 
Knowledge discovery in databases (KDD) as part of this framework indicates a process operating on distributed databases containing possible knowledge about customers. Unknown knowledge or coherence between customers and their behavior should be identified. This process aims on enhancing a customer profile without direct interaction. Knowledge discovery in databases is split up into 9 phases that can proceed as follows [11, 7]: (Phase1) Developing an understanding of the application domain and the means identifying the goal of the KDD process from the customer's viewpoint. (Phase2) Create a target data set on which discovery is to be preformed. (Phase3) Data cleaning and processing. (Phase4) Data reduction and projection. (Phase5) Matching the goals of the KDD process to data mining methods. (Phase6) Choosing the data mining algorithm(s). (Phase7) Search for patterns of interest. (Phase8) Interpreting mined patters. (Phase9) Consolidating discovered knowledge.

The recommendation process offers recommendations for customer profile enhancements. The idea behind the recommendation process includes combing wellknown methods such as rule-based-, content-based-, collaborative filtering as well as hybrid systems $[3,23,30]$ and further algorithms of different kinds. Results of the knowledge discovery process are also taken into account in order to enhance the customer profile. Whether an identified attribute is accurate or not is part of evaluation in the feedback process.

The customer profile (as defined in section 4) is generated based on information stored in databases as well as device specific information, the feedback process and information recommended by the recommender process.

Within the feedback process active and passive feedback [4] is differentiated. First the customer will be asked in e.g. monthly iterations if one of the recommended attributes is accurate or not. This is called the active feedback. The passive attempt is observing the customer's behavior after adding an additional preference that possibly leads to different services, information or products as part of the sales approach. If newly offered services are considered, possible profile enhancements are found. The result of the feedback process influences the profile generation process.

\section{Design of an Adaptive Customer Profile}

The section at hand describes the structure of an adaptive customer profile self-designed for a mobile environment. Different concepts identified by literature research $[16,2,12$, 18 ] as well as own aspects from the area of mobile commerce leads to this design shown in figure 3 . 


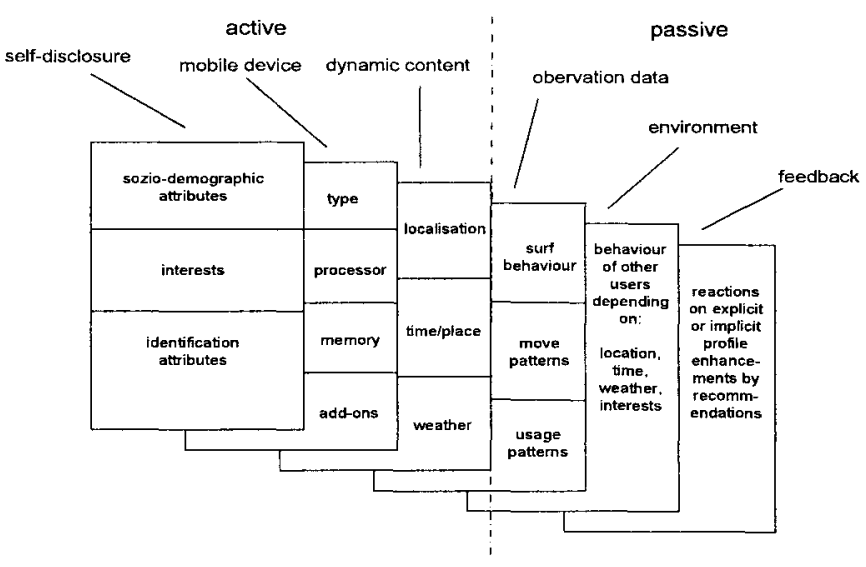

Fig. 3. Design of an adaptive customer profile

The profile consists of 6 different layers separated into 2 sections called active and passive. The active part of the customer's profile is defined as visible and accessible by the customer. In contrast, the passive part contains additional information for enhancing the active part. Information about the customer, his mobile device and dynamic situation based information is part of the active customer profile. The passive part contains observation data, information about the environment and a link to other customers in the same situation and their reaction to sales approaches.

The self-disclosure of a customer includes classical social-demographic attributes such as age, gender or interests (cinema, sports, lunch, outdoor, ..) and an identity attribute e.g. a pseudonym. The information can be stored in an $\mathrm{xml}$ similar notation as shown in the following example.

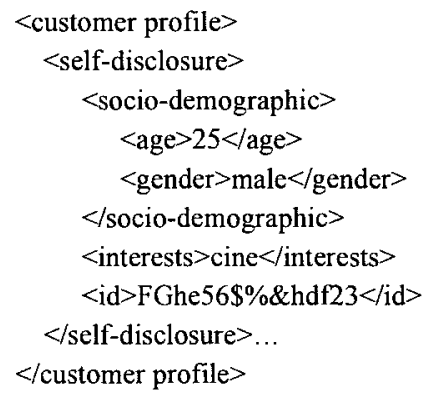

Visualizing mobile content is strongly dependent on the mobile device a customer is using. For the Playboy magazine 65 different device profiles were stored in order to guarantee the best possible display [15]. Based on a device profile, optimal graphic rendering or an adequate distribution channel (WML, XHTML, HTML, MMS or SMS) is ensured. If a device is capable of running java applications, additional features are usable. For this purpose the active customer profile should include the following attributes: device type, memory size, processor, additional enhancements. Based on the device type, information about display, colors and resolution are derivable. Enhancements a mobile device is capable of are e.g. a flash player or the java runtime environment.

$<$ customer profile>

$<$ mobile device $>$ 


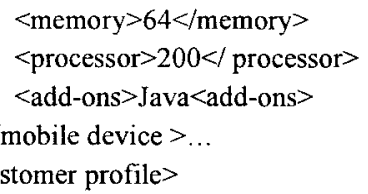

Situation dependent context information is particularly important when talking about mobile customers. The layer called dynamic content represents the current location of a customer (GPS, Cell-ID, WLAN cell) [1], time, environment (city centre, industrial zone derived by a GIS) and e.g. up-to-date weather information.

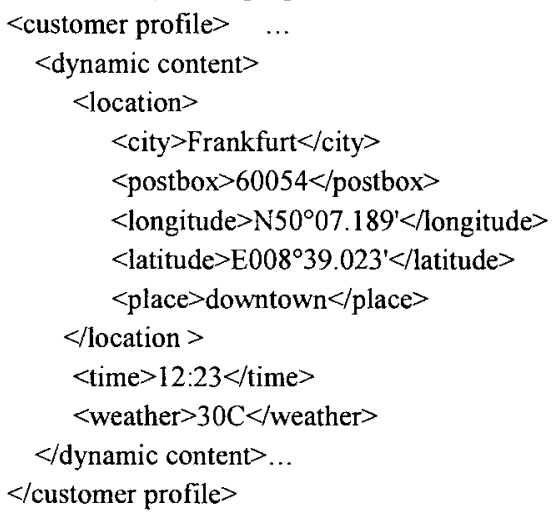

Observation data states the first information that is part of the passive profile. Observation data is information about web usage (durability, web pages sorted by categories ,...) and movement patters (which location is visited when).

Information about the environment is addressed by a separate layer of the passive profile. Hereby e.g. information about other customers at the same location and time is of importance. Amazon is a classical example from the fixed internet. A person that bought book $\mathrm{x}$, also bought $\mathrm{y}$ and might be interested in book $\mathrm{z}$. This knowledge applies to the mobile internet where a customer is maybe interested in using services others used at a specific time and place with similar profiles. Insights of this behavior, possibly derived by the process called knowledge discovery in databases, influence the recommendation of profile enhancements.

The feedback process, already described, separates the collection of insight into an active and a passive part. New profile attributes are actively communicated to the customer or questioned by the passive behavior observation.

\section{Integration in a Mobile Environment}

After discussing a framework for adaptive customer profile and a first design attempt of a customer profile, this section addresses how a customer profile can be integrated into the mobile network operator's infrastructure. Figure 4 illustrates the process within a mobile portal owned by the mobile network operator. The integration demonstrates how the necessary components are working together within the mobile operator's portal. 


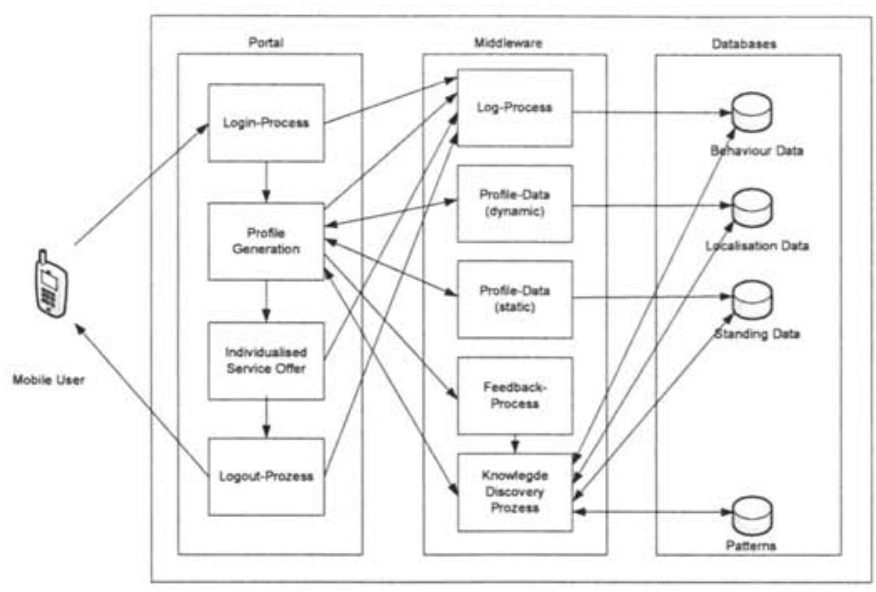

Fig. 4. Integration in a mobile environment

An already enrolled customer passes through the following process within the portal: login, profile generation, individualized service offering and logout. At every stage additional processes run in the background e.g. logging, gathering and handling dynamic and static data, feedback and knowledge discovery in databases, in order to enhance the customer profile. In the current section each stage will be discussed.

A mobile customer enters the main page of the mobile portal operated by the mobile network operator. After consent through an automatic identification process by the mobile network, a redirect is performed. A transaction pseudonym and the current location of the customer are stored in a temporal session.

The process of profile generation comprises the setup, update and storage of a customer profile. First information about the customer gathered during the login procedure (transaction pseudonym, location) is stored. Profile generation uses several sub processes (e.g. profile data as static and dynamic, feedback, knowledge discovery in databases and login), aiming at the best possible image of the customer.

Based on an always up-to-date customer profile, individualized services can be offered [8], independent of whether the idea of an advertising-based revenue model [10] is being followed or not. The baseline of every individualized service offering is an image of a customer.

The logout process finalizes the service usage. Every transaction is closed, log information is stored and potential payments are allocated.

The collection of static customer information as a transaction pseudonym, age and sex are part of the process as well as static mid-term information as hobbies (see section 4). Dynamic information is given by point of interests the customer visited, date, time or residence time. All data is pseudonymously stored in databases.

The logging process observes the following sub processes: login (date, time, location of the customer, mobile device, and pseudonym), profile generation (setup and updates), individualized service offering (service offer, service usage, usage duration, interaction level) and logout. Gathered information is pseudonymously stored in a database.

Knowledge discovery in databases is a process separated into 9 sub processes already discussed in section 4. Applied on several distributed databases, so far undiscovered insights should lead to customer profile enhancements without direct interaction.

The feedback process was already described in section 4 . 


\section{Data Protection}

Profiling customers as discussed within the paper proves difficult because it implies knowledge in the area of data protection. While profiling the customer no personal information is stored in databases. Furthermore, the localization is only performed after consent from the customer. Every information disclosure accords to the EU directive $95 / 46 / \mathrm{EC}$ on protection of individuals with regard to the processing of personal data and free movement of such data.

\section{Conclusion and Outlook}

The paper at hand discussed the problem of information overflow on the one hand and individualized sales approaches in order to increase mobile data communication usage on the other hand, in a mobile market described by a high penetration, competition and the absence of differentiation. Technical as well as conceptual approaches for individualization sales approaches were presented based on adaptive customer profiles. Precise customer profiles are the baseline of accurate recommendations. A proposal of a framework to design adaptive customer profiles, the construction of a customer profile for a mobile environment and a discussion on how to integrate such a profile into a platform of the mobile network operator, was part of the paper. An advertising-based revenue model [10] is able to match advertiser and customers in a better way than before.

The enhancement of the framework, the embodying of the customer profile in a mobile environment and its integration validated by the architecture analysis and a case study including complexity, hardware, response time, benefit analysis, as well as an implementation as a prototype are planned. Further discussions on data protection are necessary. According the research approach based on design research in section 2 , the work this paper presented covers the first and the second stage (awareness of a problem and suggestions).

\section{References}

41. 1. A. Albers, S. Figge and M. Radmacher, LOC3 - Architecture Proposal for Efficient Subscriber Localisation in Mobile Commerce Infrastructures, Proceedings of 2nd IEEE International Workshop on Mobile Commerce and Service, Munich, Germany, 2005.

42. 2. G. Amato and U. Straccia, User Profile Modelling and Applications to Digital Libraries, ECDL '99, Abitcboul, S., Vercoustre, A.-M.(Eds.), 1999, pp. 184-197.

43. 3. M. Balabanović, and Y. Shoham, Fab: content-based, collaborative recommendation, Communication of the ACM, March 1997/Vol. 40, No. 3.

44. 4. R. Bulander, M. Decker, B. Kölmel and G. Schiefer, Kontextsensitives mobiles Marketing, in: B. König-Ries, M. Klein (Hrsg.): Mobile Datenbanken und Informationssysteme, in Business, Technologie und Web. BTW 2005, Universität Karlsruhe 2005, pp. 11-20.

45. 5. Bundesnetzagentur, 2006, Jahresbericht 2006. Bundesnetzagentur, Bonn.

46. 6. R. Carreira, J.M. Crato, D. Gonçalves and J.A. Jorge, Evaluating Adaptive User Profiles for News Classification, 2004.

47. 7. U. Fayyad, G. Piatetsky-Shapiro and P. Smyth, Knowledge Discovery and Data Mining Towards a Unifying Framework, 1996. 
48. 8. S. Figge, Situation-dependent services-a challenge for mobile network operators, Journal of Business Research, Volume 57, Issue 12, 2004, pp. 1416-1422.

49. 9. S. Figge and A. Albers, Individualising M-Commerce Services by Semantic User Situation Modelling, Proceedings of the 7 th International Conference Wirtschaftsinformatik, Bamberg, 2005.

50. 10. S. Figge and S. Theysohn, Quantifizierung IKS-basierter Marktleistungen Analyse eines werbefinanzierten Geschäftsmodells für den Mobile Commerce, Wirtschafsinformatik 48 (2), 2006, pp. 96-106.

51. 11. W.J. Frawley, G. Piatetsky-Shapiro and C.J. Matheus, Knowledge Discovery in Databases: An Overview. AAAI, 1992, pp. 56-70.

52. 12. M. Goel, S. Sarkar, Web Site Personalization Using User Profile Information, AH2002, P. De Bra, P. Brusilovsky and R. Concjo, (Eds.), 2002, pp. 510-513.

53. 13. D. Goren-Bar and O. Glinansky, FIT-recommending TV programs to family members. Computer \& Graphics, 28, 2004, pp.149-156.

54. 14. A.R. Hevner, S.T. March, J. Park and S. Ram, Design Science Information Systems Research. MIS Quarterly, Vol. 28 No. 1, 2004, pp. 75-105.

55. 15. C. Kaspar, O. von Wersch, I. Hochstatter, A. Albers, S. Figge, et al., Mobile Anwendungen - Best Practices in der TIME-Branche. Hrsg.: T. Hess, S. Hagenhoff, D. Hogrefe, C. Linnhoff-Popien, K. Rannenberg and F. Straube, Universitätsverlag Göttingen, 2005.

56. 16. R.R. Korfhage, Query Enhancement by User Profiles, Joint BCS \& ACM Symposium on Research \& Development in Information Retrieval, 1984, pp.111-121.

57. 17. R. Kraemer and P. Schwander, Bluetooth based wireless Internet applications for indoor hot spots: experience, 2000.

58. 18. R.J. Lukose, E. Adar, J.R. Tyler and C. Sengupta, SHOCK: Communicating with Computational messages and Automatic Private Profiles, Proceedings of WWW 2003, May 20-24, Budapest, 2003, pp. 291-300.

59. 19. B. Salem and M. Rauterberg, Multiple User Profile Merging (MUPE): Key Challenges for Environment Awareness, EUSAI 2004, pp. 196-206.

60.20 . J. Schackmann, Ökonomische vorteilhafte Individualisierung und Personalisierung, 2002

61. 21. M. Schuhmann, Individualität und Produktindividualisierung - Kundenprofile für die Personalisierung von Produkten, 2004.

62. 22. C. Shapiro and H.R. Varian, Information Rules, 1999.

63. 23. S. Singh, M. Shepherd, J. Duffy and C. Watters, An Adaptive User Profile for Filtering News Based on a User Interest Hierarchy, 2006.

64. 24. U. Spitzer, Recommender Systeme im E-Commerce. Wirtschaftsuniversität Wien, 2005.

65. 25. K. Sugiyama, K. Hatano and M. Yoshikawa, Adaptive Web Search Based on User Profile Constructed without Any Effort from Users, 2005.

66. 26. P. Srinil, and O. Pinngern, Adaptive User Profile for Information Retrieval from the Web, 2002.

67. 27. H. Takeda, P. Veerkamp, T. Tomiyama and H. Yoshikawam, Modeling Design Processes, AI Magazine, 1990, pp. 37-48.

68. 28. V. Vaishnavi and B. Kuechler, Design Research in Information Systems, AIS 2006.

69. 29. Vodafone Group Plc, Key Performance Indicators, 2006, Available:

70. http://www.vodafone.com/assets/files/en/VOD_KPIs_20041231_2.xls, Abruf am 2005-11-01

71. 30. Y.Z. Wei, L. Moreau and N.R. Jennings, A Market-Based Approach to Recommender Systems, ACM Transactions on Information Systems (TOIS), 2005, pp. $227-266$.

72. 31. P. Zipkin, The limits of mass customization, Sloan Management Review 42,3, 2001, pp. 81-87. 